Bull. Korean Math. Soc. 52 (2015), No. 5, pp. 1753-1757

http://dx.doi.org/10.4134/BKMS.2015.52.5.1753

\title{
A REMARK ON UNIQUE CONTINUATION FOR THE CAUCHY-RIEMANN OPERATOR
}

\author{
IHYEOK SEO
}

\begin{abstract}
In this note we obtain a unique continuation result for the differential inequality $|\bar{\partial} u| \leq|V u|$, where $\bar{\partial}=\left(i \partial_{y}+\partial_{x}\right) / 2$ denotes the Cauchy-Riemann operator and $V(x, y)$ is a function in $L^{2}\left(\mathbb{R}^{2}\right)$.
\end{abstract}

\section{Introduction}

The unique continuation property is one of the most interesting properties of holomorphic functions $f \in H(\mathbb{C})$. This property says that if $f$ vanishes in a non-empty open subset of $\mathbb{C}$, then it must be identically zero. Note that $u \in C^{1}\left(\mathbb{R}^{2}\right)$ satisfies the Cauchy-Riemann equation $\left(i \partial_{y}+\partial_{x}\right) u=0$ if and only if it defines a holomorphic function $f(x+i y) \equiv u(x, y)$ on $\mathbb{C}$. From this point of view, one can see that a $C^{1}$ function satisfying the equation has the unique continuation property.

In this note we consider a class of non-holomorphic functions $u$ which satisfy the differential inequality

$$
|\bar{\partial} u| \leq|V u|,
$$

where $\bar{\partial}=\left(i \partial_{y}+\partial_{x}\right) / 2$ denotes the Cauchy-Riemann operator and $V(x, y)$ is a function on $\mathbb{R}^{2}$.

The best positive result for (1.1) is due to Wolff [9] (see Theorem 4 there) who proved the property for $V \in L^{p}$ with $p>2$. On the other hand, there is a counterexample [8] to unique continuation for (1.1) with $V \in L^{p}$ for $p<2$. The remaining case $p=2$ seems to be unknown for the differential inequality (1.1), and note that $L^{2}$ is a scale-invariant space of $V$ for the equation $\bar{\partial} u=V u$. Here we shall handle this problem. Our unique continuation result is the following theorem which is based on bounds for a Fourier multiplier from $L^{p}$ to $L^{q}$.

Theorem 1.1. Let $1<p<2<q<\infty$ and $1 / p-1 / q=1 / 2$. Assume that $u \in L^{p} \cap L^{q}$ satisfies the inequality (1.1) with $V \in L^{2}$ and vanishes in a non-empty open subset of $\mathbb{R}^{2}$. Then it must be identically zero.

Received January 20, 2015.

2010 Mathematics Subject Classification. Primary 35B60, 35F05.

Key words and phrases. unique continuation, Cauchy-Riemann operator. 
The unique continuation property also holds for harmonic functions, which satisfy the Laplace equation $\Delta u=0$, since they are real parts of holomorphic functions. This was first extended by Carleman [1] to a class of non-harmonic functions satisfying the inequality $|\Delta u| \leq|V u|$ with $V \in L^{\infty}\left(\mathbb{R}^{2}\right)$. There is an extensive literature on later developments in this subject. In particular, the problem of finding all the possible $L^{p}$ functions $V$, for which $|\Delta u| \leq|V u|$ has the unique continuation, is completely solved (see $[3,5,7]$ ). See also the survey papers of Kenig [4] and Wolff [10] for more details, and the recent paper of Kenig and Wang [6] for a stronger result which gives a quantitative form of the unique continuation.

Throughout the paper, the letter $C$ stands for positive constants possibly different at each occurrence. Also, the notations $\widehat{f}$ and $\mathcal{F}^{-1}(f)$ denote the Fourier and the inverse Fourier transforms of $f$, respectively.

\section{A preliminary lemma}

The standard method to study the unique continuation property is to obtain a suitable Carleman inequality for relevant differential operator. This method originated from Carleman's classical work [1] for elliptic operators. In our case we need to obtain the following inequality for the Cauchy-Riemann operator $\bar{\partial}=\left(i \partial_{y}+\partial_{x}\right) / 2$, which will be used in the next section for the proof of Theorem 1.1:

Lemma 2.1. Let $f \in C_{0}^{\infty}\left(\mathbb{R}^{2} \backslash\{0\}\right)$. For all $t>0$, we have

$$
\left\||z|^{-t} f\right\|_{L^{q}} \leq C\left\||z|^{-t} \bar{\partial} f\right\|_{L^{p}}
$$

if $1<p<2<q<\infty$ and $1 / p-1 / q=1 / 2$. Here, $z=x+i y \in \mathbb{C}$ and $C$ is a constant independent of $t$.

Proof. First we note that

$$
\bar{\partial}\left(z^{-t} f\right)=z^{-t} \bar{\partial} f+f \bar{\partial}\left(z^{-t}\right)=z^{-t} \bar{\partial} f
$$

for $z \in \mathbb{C} \backslash\{0\}$. Then the inequality (2.1) is equivalent to

$$
\left\|z^{-t} f\right\|_{L^{q}} \leq C\left\|\bar{\partial}\left(z^{-t} f\right)\right\|_{L^{p}} .
$$

By setting $g=z^{-t} f$, we are reduced to showing that

$$
\|g\|_{L^{q}} \leq C\left\|\left(i \partial_{y}+\partial_{x}\right) g\right\|_{L^{p}}
$$

for $g \in C_{0}^{\infty}\left(\mathbb{R}^{2} \backslash\{0\}\right)$. To show this, let us first set

$$
\left(i \partial_{y}+\partial_{x}\right) g=h,
$$

and let $\psi_{\delta}: \mathbb{R}^{2} \rightarrow[0,1]$ be a smooth function such that $\psi_{\delta}=0$ in the ball $B(0, \delta)$ and $\psi_{\delta}=1$ in $\mathbb{R}^{2} \backslash B(0,2 \delta)$. Then, using the Fourier transform in $(2.2)$, we see that

$$
(-\eta+i \xi) \widehat{g}(\xi, \eta)=\widehat{h}(\xi, \eta) .
$$


Thus, by Fatou's lemma we are finally reduced to showing the following uniform boundedness for a multiplier operator having the multiplier $m(\xi, \eta)=$ $\psi_{\delta}(\xi, \eta) /(-\eta+i \xi)$ :

$$
\left\|\mathcal{F}^{-1}\left(\frac{\psi_{\delta}(\xi, \eta) \widehat{h}(\xi, \eta)}{-\eta+i \xi}\right)\right\|_{L^{q}} \leq C\|h\|_{L^{p}}
$$

uniformly in $\delta>0$.

From now on, we will show (2.3) using Young's inequality for convolutions and Littlewood-Paley theorem $([2])$. Let us first set for $k \in \mathbb{Z}$

$$
\widehat{T h}(\xi, \eta)=m(\xi, \eta) \widehat{h}(\xi, \eta) \text { and } \widehat{T_{k} h}(\xi, \eta)=m(\xi, \eta) \chi_{k}(\xi, \eta) \widehat{h}(\xi, \eta),
$$

where $\chi_{k}(\cdot)=\chi\left(2^{k} \cdot\right)$ for $\chi \in C_{0}^{\infty}\left(\mathbb{R}^{2}\right)$ which is such that $\chi(\xi, \eta)=1$ if $|(\xi, \eta)| \sim$ 1 , and zero otherwise. Also, $\sum_{k} \chi_{k}=1$. Now we claim that

$$
\left\|T_{k} h\right\|_{L^{q}} \leq C\|h\|_{L^{p}}
$$

uniformly in $k \in \mathbb{Z}$. Then, since $1<p<2<q<\infty$, by the Littlewood-Paley theorem together with Minkowski's inequality, we get the desired inequality (2.3) as follows:

$$
\begin{aligned}
\left\|\sum_{k} T_{k} h\right\|_{L^{q}} & \leq C\left\|\left(\sum_{k}\left|T_{k} h\right|^{2}\right)^{1 / 2}\right\|_{L^{q}} \\
& \leq C\left(\sum_{k}\left\|T_{k} h\right\|_{L^{q}}^{2}\right)^{1 / 2} \\
& \leq C\left(\sum_{k}\left\|h_{k}\right\|_{L^{p}}^{2}\right)^{1 / 2} \\
& \leq C\left\|\left(\sum_{k}\left|h_{k}\right|^{2}\right)^{1 / 2}\right\|_{L^{p}} \\
& \leq C\left\|\sum_{k} h_{k}\right\|_{L^{p}},
\end{aligned}
$$

where $h_{k}$ is given by $\widehat{h_{k}}(\xi, \eta)=\chi_{k}(\xi, \eta) \widehat{h}(\xi, \eta)$. Now it remains to show the claim (2.4). But, this follows easily from Young's inequality. Indeed, note that

$$
T_{k} h=\mathcal{F}^{-1}\left(\frac{\psi_{\delta}(\xi, \eta) \chi_{k}(\xi, \eta)}{-\eta+i \xi}\right) * h
$$

and by Plancherel's theorem

$$
\begin{aligned}
\left\|\mathcal{F}^{-1}\left(\frac{\psi_{\delta}(\xi, \eta) \chi_{k}(\xi, \eta)}{-\eta+i \xi}\right)\right\|_{L^{2}} & =\left\|\frac{\psi_{\delta}(\xi, \eta) \chi_{k}(\xi, \eta)}{-\eta+i \xi}\right\|_{L^{2}} \\
& \leq C\left(\int_{|(\xi, \eta)| \sim 2^{-k}} \frac{1}{\eta^{2}+\xi^{2}} d \xi d \eta\right)^{1 / 2} \\
& \leq C .
\end{aligned}
$$


Since we are assuming the gap condition $1 / p-1 / q=1 / 2$, by Young's inequality for convolutions, this readily implies that

$$
\left\|T_{k} h\right\|_{L^{q}} \leq\left\|\mathcal{F}^{-1}\left(\frac{\psi_{\delta}(\xi, \eta) \chi_{k}(\xi, \eta)}{-\eta+i \xi}\right)\right\|_{L^{2}}\|h\|_{L^{p}} \leq C\|h\|_{L^{p}}
$$

as desired.

\section{Proof of Theorem 1.1}

The proof is standard once one has the Carleman inequality (2.1) in Lemma 2.1 .

Without loss of generality, we may show that $u$ must vanish identically if it vanishes in a sufficiently small neighborhood of zero. Then, since we are assuming that $u \in L^{p} \cap L^{q}$ vanishes near zero, from (2.1) with a standard limiting argument involving a $C_{0}^{\infty}$ approximate identity, it follows that

$$
\left\||z|^{-t} u\right\|_{L^{q}} \leq C\left\||z|^{-t} \bar{\partial} u\right\|_{L^{p}} .
$$

Thus by (1.1) we see that

$$
\left\||z|^{-t} u\right\|_{L^{q}(B(0, r))} \leq C\left\||z|^{-t} V u\right\|_{L^{p}(B(0, r))}+C\left\||z|^{-t} \bar{\partial} u\right\|_{L^{p}\left(\mathbb{R}^{2} \backslash B(0, r)\right)},
$$

where $B(0, r)$ is the ball of radius $r>0$ centered at 0 . Then, using Hölder's inequality with $1 / p-1 / q=1 / 2$, the first term on the right-hand side in the above can be absorbed into the left-hand side as follows:

$$
\begin{aligned}
C\left\||z|^{-t} V u\right\|_{L^{p}(B(0, r))} & \leq C\|V\|_{L^{2}(B(0, r))}\left\||z|^{-t} u\right\|_{L^{q}(B(0, r))} \\
& \leq \frac{1}{2}\left\||z|^{-t} u\right\|_{L^{q}(B(0, r))}
\end{aligned}
$$

if we choose $r$ small enough. Here, $\left\||z|^{-t} u\right\|_{L^{q}(B(0, r))}$ is finite since $u \in L^{q}$ vanishes near zero. Hence we get

$$
\begin{aligned}
\left\|(r /|z|)^{t} u\right\|_{L^{q}(B(0, r))} & \leq 2 C\|\bar{\partial} u\|_{L^{p}\left(\mathbb{R}^{2} \backslash B(0, r)\right)} \\
& \leq 2 C\|V\|_{L^{2}}\|u\|_{L^{q}} \\
& <\infty
\end{aligned}
$$

By letting $t \rightarrow \infty$, we now conclude that $u=0$ on $B(0, r)$. This implies $u \equiv 0$ by a standard connectedness argument.

Acknowledgment. I would like to thank Jenn-Nan Wang for pointing out a preprint ([6]) and for some comments.

\section{References}

[1] T. Carleman, Sur un problème d'unicité pour les systèmes d'équations aux derivées partielles à deux variables indépendantes, Ark. Mat. Astr. Fys. 26 (1939), no. 17, 1-9.

[2] L. Grafakos, Classical Fourier Analysis, Springer, New York, 2008.

[3] D. Jerison and C. E. Kenig, Unique continuation and absence of positive eigenvalues for Schrödinger operators, Ann. of Math. 121 (1985), no. 3, 463-494. 
[4] C. E. Kenig, Restriction theorems, Carleman estimates, uniform Sobolev inequalities and unique continuations, Harmonic analysis and partial differential equations (El Escorial, 1987), 69-90, Lecture Notes in Math. 1384, Springer, Berlin, 1989.

[5] C. E. Kenig and N. Nadirashvili, A counterexample in unique continuation, Math. Res. Lett. 7 (2000), no. 5-6, 625-630.

[6] C. E. Kenig and J.-N. Wang, Quantitative uniqueness estimates for second order elliptic equations with unbounded drift, Math. Res. Lett. 22 (2015), no. 4, 1159-1175.

[7] H. Koch and D. Tataru, Sharp counterexamples in unique continuation for second order elliptic equations, J. Reine Angew. Math. 542 (2002), 133-146.

[8] N. Mandache, A counterexample to unique continuation in dimension two, Comm. Anal. Geom. 10 (2002), no. 1, 1-10.

[9] T. H. Wolff, A property of measures in $\mathbb{R}^{n}$ and an application to unique continuation, Geom. Funct. Anal. 2 (1992), no. 2, 225-284.

[10] — Recent work on sharp estimates in second-order elliptic unique continuation problems, J. Geom. Anal. 3 (1993), no. 6, 621-650.

Department of Mathematics

SUNGKYUNKWAN UNIVERSITY

Suwon 440-746, Korea

E-mail address: ihseo@skku.edu 